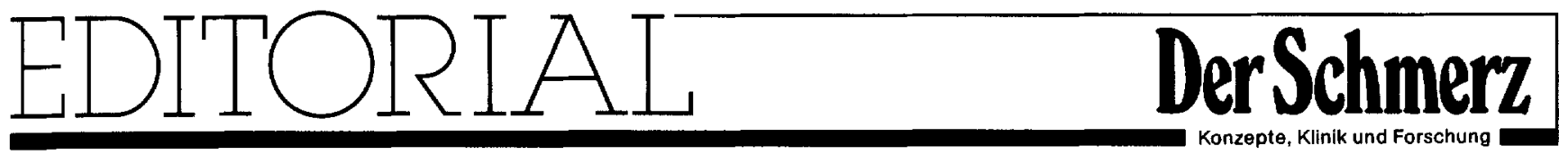

\title{
Akute Schmerzbehandlung in operativer und konservativer Medizin
}

\author{
W. Dick \\ Klinik für Anästhesiologie der Universität Mainz
}

Im letzten, diesem und den folgenden Heften will die Zeitschrift Der Schmerz einen Themenkomplex zusammenfassend darstellen, der immer mehr Bedeutung im klinischen wie außerklinischen Bereich gewinnt, die Behandlung des akuten Schmerzes in der konservativen und operativen Medizin.

Nach wie vor müssen niedergelassene Kollegen und Klinikärzte davon ausgehen, daß die überwiegende Mehrzahl ihrer Patienten die Behandlung eines akuten Schmerzzustandes, sei es in einer Notsituation, einem Notfall oder in der perioperativen Phase, als unbefriedigend empfinden.

Dazu tragen oft mangelnde Kenntnis der geeigneten Methoden und Mittel zur Analgesie, Verunsicherungen hinsichtlich bestimmter Präparate durch wenig seriöse „Fachblätter“ (z.B. Metamizol) bei, aber auch ein häufig zu oberflächliches Eingehen auf die Schmerzsituation des Patienten, oder gar eine Fehleinschätzung der Schmerzintensität durch Arzt und nichtärztliches medizinisches Personal (M. Forrest, G. Hermann und B. Andersen (1989) Assessment of pain : a comparison between patients and doctors: Acta Anaesthesiol Scand 33:255).

Zusätzlich wird gerade die perioperative Schmerztherapie dadurch an Bedeutung gewinnen, da $B$ ambulantes Operieren nicht nur in Praxisambulanzen, sondern auch in der Klinik Popularität gewinnt. Aus Furcht vor unerwünschten - weil unkontrollierbaren - Nebenwirkungen eines Analgetikums in der postoperativen Phase nach der Entlassung des Patienten, wird vermutlich nicht selten von jeglicher Schmerzmedikation Abstand genommen.

Mit dieser zusammengefaßten Darstellung der wichtigsten Probleme der akuten Schmerzbehandlung wollen wir dazu beitragen, die Möglichkeiten der Schmerztherapie effektiver und sicherer zu nutzen.

Wir haben das Spektrum der Darstellungen bewußt mit einigen neueren Erkenntnissen (z.B. periphere Opiatrezeptoren) begonnen, um die Ausgangsbasis für eine wirksame und rationelle, dabei auch ökonomische Schmerztherapie zu begründen.

Eine Zusammenstellung (Seeling et al.) beschäftigt sich zudem kritisch mit der Frage, ob Schmerztherapie neben ihrem zweifellos humanitären und psychologisch wirkungsvollen Charakter überhaupt einen nachgewiesen positiven Einfluß auf einen Krankheitsverlauf hat.

Sodann werden die einzelnen Bereiche der akuten Schmerztherapie von kompetenten Autoren ausführlich abgehandelt, z.B. Notfallsituationen wie Koliken und sonstige gastrointestinale Schmerzen, die Palette der peri-, intra- und postoperativen Schmerztherapie, die häufig für eine inadäquate Behandlung als Grund reklamierten vorgeblichen Suchtgefahren, aber auch Analgesie unter der Geburt sowie in der operativen wie nichtoperativen Behandlung des Kindes.

Wir hoffen, mit der Abhandlung eines geschlossenen Problemkreises, in mehreren Heften der Zeitschrift ein echtes Informationsbedürfnis der Leser aufgegriffen zu haben.

Wie stets sind wir für konstruktive, kritische Rückäußerungen dankbar.

Prof. Dr. W. Dick

Klinik für Anästhesiologie

Johannes-Gutenberg-Universität

Langenbeckstraße 1

W-6500 Mainz 